\title{
Extended Continuous Infusion of Methylene Blue for Refractory Septic Shock
}

\author{
Abhishek Jaiswal ${ }^{1}$, Manish Kumar ${ }^{2}$, Elizabeth Silver ${ }^{3}$
}

\begin{abstract}
Objective: Highlight the utility of methylene blue (MTB) infusion for extended period for refractory vasoplegia.

Introduction: Hypotension refractory to vasopressor therapy in sepsis is associated with high mortality and limited therapeutic options. Dysregulated nitric oxide (NO) pathway seems to be a major driver, and, therefore, MTB, which inhibits inducible NO synthase activity and decreases cyclic guanosine monophosphate (GMP) accumulation by directly competing with NO by binding to soluble guanylyl cyclase, has been explored.

Case description: We describe a successful reversal of refractory septic shock with prolonged MTB infusion in a patient supported on multiple vasopressors at the highest clinical doses as well as venovenous extracorporeal membrane oxygenation (VV-ECMO).

Conclusion and clinical significance: Current report suggests a potential role of MTB infusion in refractory vasoplegia even in advanced vasoplegic shock.

Keywords: Extra corporeal membrane oxygenation, Methylene blue, Refractory shock, Sepsis induced.

Indian Journal of Critical Care Medicine (2020): 10.5005/jp-journals-10071-23376
\end{abstract}

\section{BACKGROUND}

Patients in septic shock refractory to vasoactive agents have limited therapeutic options with poor prognosis. Dysregulated nitric oxide (NO) pathway seems to be a major driver, and, therefore, methylene blue (MTB), which inhibits inducible NO synthase activity and decreases cyclic guanosine monophosphate (GMP) accumulation by directly competing with NO by binding to soluble guanylyl cyclase, has been explored. ${ }^{1-5}$ We describe a successful reversal of refractory septic shock with prolonged MTB infusion in a patient supported on multiple vasopressors at the highest clinical doses as well as venovenous extracorporeal membrane oxygenation (VV-ECMO).

\section{Case Description}

A 38-year-old male was brought to our hospital for worsening lethargy and hypoxia. Initial assessment showed afebrile, lethargic male with tachypnea, bibasilar inspiratory crackles on chest auscultation, and hypoxia which improved on $15 \mathrm{~L} /$ minute oxygen supplementation. Investigations revealed metabolic acidosis $(\mathrm{pH}-$ 6.95), blood sugar $>1000 \mathrm{mg} / \mathrm{dL}$, an anion gap of 31, and bibasilar infiltrates on chest imaging. Patient was started on intravenous (IV) fluid and insulin infusions, placed on noninvasive ventilation, and antibiotics. However, worsening hypoxia and breathing led to endotracheal intubation. Shortly thereafter, he became hypotensive, requiring boluses of IV fluid and vasopressor infusions (Fig. 1). His antibiotics were broadened and continuous venovenous renal replacement (CVVH) therapy was initiated for worsening metabolic acidosis, oliguria, and escalating the requirement of vasopressors. However, within 2 hours, he had a pulse electrical activity cardiac arrest. Bedside transthoracic echocardiogram showed hyper dynamic left ventricle without any pericardial effusion.

At 25 hours from presentation, and 4 hours after cardiac arrest, the patient was started on VV-ECMO support for ongoing

\footnotetext{
${ }^{1-3}$ Department of Cardiology, Hartford Hospital Heart and Vascular Institute, Hartford, Connecticut, USA

Corresponding Author: Abhishek Jaiswal, Department of Cardiology, Hartford Hospital Heart and Vascular Institute, Hartford, Connecticut, USA, Phone: +1 8609721212, e-mail: Abhishek.Jaiswal@hhchealth.org How to cite this article: Jaiswal A, Kumar M, Silver E. Extended Continuous Infusion of Methylene Blue for Refractory Septic Shock. Indian J Crit Care Med 2020;24(3):206-207.

Source of support: Nil

Conflict of interest: None
}

hypoxia, inability to undergo CVVH due to hypotension. Continued hypotension despite improving hypoxia and acidosis with ECMO support along with multiple vasopressors prompted a trial of MTB.

Following an IV bolus of MTB (172 mg), an infusion was started at $0.51 / \mathrm{mg} / \mathrm{kg} / \mathrm{hour}$ for next 10 hours, resulting in improved blood pressure, reinitiation of $\mathrm{CVVH}$, and decreased vasopressor requirement. Post infusion patient maintained hemodynamics on norepinephrine at $36 \mu \mathrm{g} /$ minute, epinephrine at $0.25 \mu \mathrm{g} / \mathrm{kg} /$ minute, and vasopressin at 0.07 units/minute with improved urine output resulting in discontinuation of $\mathrm{CVVH}$. However, 22 hours into MTB infusion discontinuation, he developed fever and hypotension prompting escalation of vasopressors infusions; and, due to continued hypotension despite maximum dose of multiple vasopressors, the MTB infusion was restarted with subsequent hemodynamic improvement. He was found to have Klebsiella pneumonia bacteremia and antibiotics were tailored per sensitivities. The MTB infusion was stopped after 54 hours and patient was off all vasopressors in next 6-7 days and eventually was discharged.

() The Author(s). 2020 Open Access This article is distributed under the terms of the Creative Commons Attribution 4.0 International License (https://creativecommons. org/licenses/by-nc/4.0/), which permits unrestricted use, distribution, and non-commercial reproduction in any medium, provided you give appropriate credit to the original author(s) and the source, provide a link to the Creative Commons license, and indicate if changes were made. The Creative Commons Public Domain Dedication waiver (http://creativecommons.org/publicdomain/zero/1.0/) applies to the data made available in this article, unless otherwise stated. 

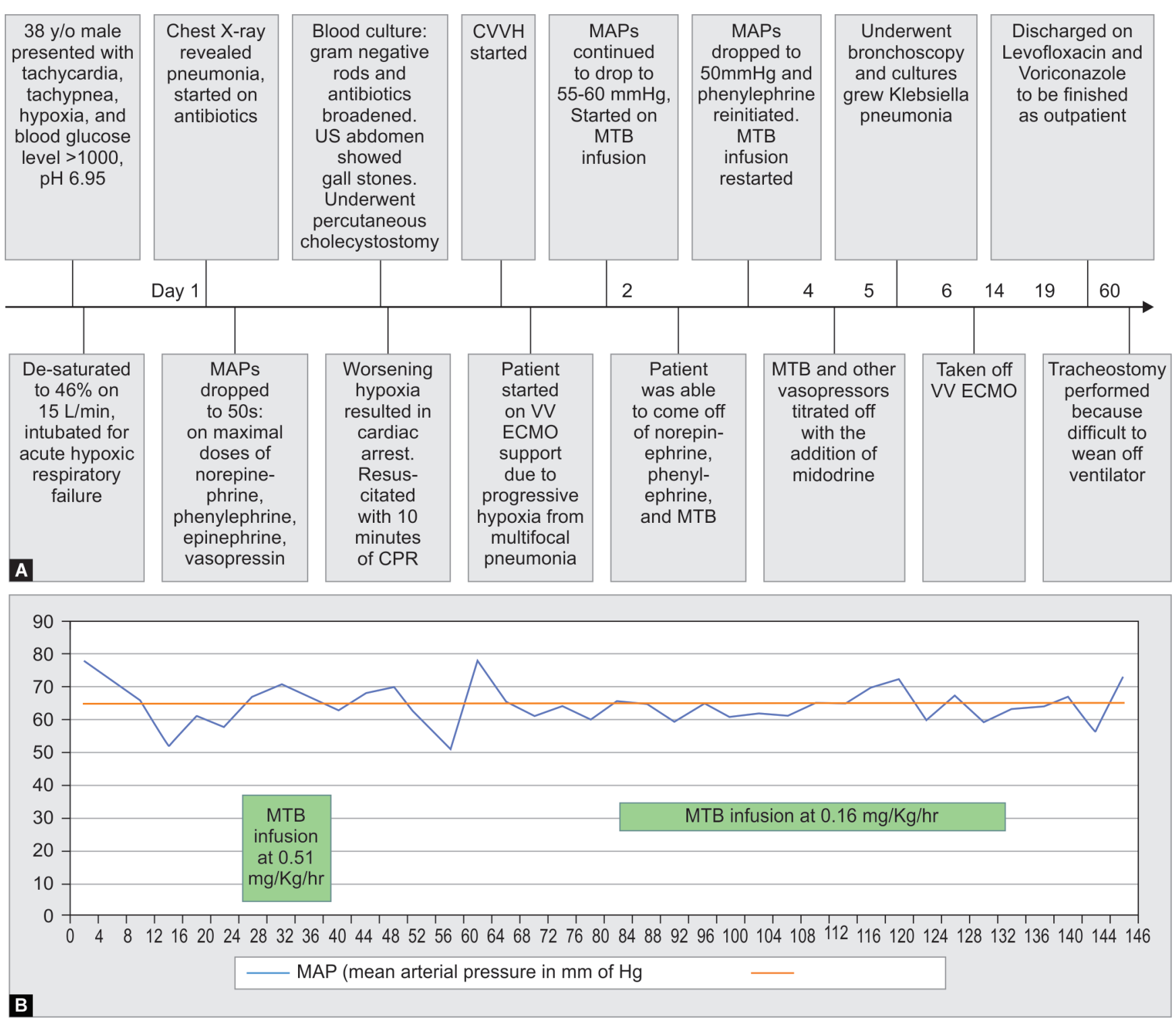

Figs $1 \mathrm{~A}$ and B: (A) Timeline of the patient's significant events in the hospital from admission through discharge; (B) Effects of methylene blue on mean arterial pressures (MAPs) (arrows representing single-dose administration)

\section{Discussion}

Prolonged MTB infusion reduced vasopressor requirement, improved the overall hemodynamics and might have contributed to the survival of our patient with refractory septic shock. A successful experience with prolonged MTB infusion ( 44 hours) was reported in a less sicker patient requiring only modest doses of vasopressors, ${ }^{2}$ whereas our patient was far sicker with renal failure and refractory hypotension, despite multiple vasopressor infusions at maximal doses, correction of hypoxia, and acidosis with ongoing ECMO support.

\section{Conclusion and Clinical Significance}

Current report highlights the potential role of MTB infusion in refractory vasoplegia even in advanced septic shock.

\section{References}

1. Preiser JC, Lejeune P, Roman A, Carlier E, De Backer D, Leeman M, et al. Methylene blue administration in septic shock: a clinical trial. Crit Care Med 1995;23(2):259-264. DOI: 10.1097/00003246-199502000-00010.

2. Brown G, Frankl D, Phang T. Continuous infusion of methylene blue for septic shock. Postgrad Med J 1996;72(852):612-614. DOI: 10.1136/ pgmj.72.852.612.

3. Paciullo CA, McMahon Horner D, Hatton KW, Flynn JD. Methylene blue for the treatment of septic shock. Pharmacotherapy 2010;30(7):702715. DOI: 10.1592/phco.30.7.702.

4. Kwok ES, Howes D. Use of methylene blue in sepsis: a systematic review. J Intensive Care Med 2006;21(6):359-363. DOI: 10.1177/0885066606290671.

5. Belletti A, Musu M, Silvetti S, Saleh O, Pasin L, Monaco F, et al. Nonadrenergic vasopressors in patients with or at risk for vasodilatory shock. A systematic review and meta-analysis of randomized trials. PLoS One 2015;10(11):e0142605. DOI: 10.1371/journal.pone.0142605. 